\title{
Bladder Inflammatory Myofibroblastic Tumor
}

National Cancer Institute

\section{Source}

National Cancer Institute. Bladder Inflammatory Myofibroblastic Tumor. NCI Thesaurus.

Code 66177.

A multinodular intermediate fibroblastic neoplasm arising from the bladder. It is characterized by the presence of spindle-shaped fibroblasts and myofibroblasts, and a chronic inflammatory infiltrate composed of eosinophils, lymphocytes and plasma cells. 